\title{
A multi-pathway model for Photosynthetic reaction center
}

\author{
M. Qin ${ }^{1,2}$, H. Z Shen ${ }^{1,2}$, and X. X. Yi ${ }^{2} *$ \\ ${ }^{1}$ School of Physics and Optoelectronic Technology \\ Dalian University of Technology, Dalian 116024, China \\ ${ }^{2}$ Center for Quantum Sciences and School of Physics, \\ Northeast Normal University, Changchun 130024, China
}

\begin{abstract}
Charge separation in light-harvesting complexes occurs in a pair of tightly coupled chlorophylls at the heart of photosynthetic reaction centers of both plants and bacteria. Recently it has been shown that quantum coherence can, in principle, enhance the efficiency of a solar cell, working like a quantum heat engine (QHE). Here, we propose a biological quantum heat engine (BQHE) motivated by Photosystem II reaction center (PSII RC) to describe the charge separation. Our model mainly considers two charge-separation pathways more than that in the published literature. The two pathways can interfere via cross-couplings and work together to enhance the charge-separation yields. We explore how these cross-couplings increase the current and voltage of the charge separation and discuss the advantages of multiple pathways in terms of current and power. The robustness of the $\mathrm{BQHE}$ against the charge recombination in natural PSII RC and dephasing induced by environments is also explored, and extension from two pathways to multiple pathways is made. These results suggest that naturemimicking architectures with engineered multiple pathways for charge separations might be better for artificial solar energy devices.
\end{abstract}

PACS numbers: $42.50 . \mathrm{Gy}, 42.50 . \mathrm{Nn}, 84.60 . \mathrm{Jt}, 82.39 . \mathrm{Jn}$

\section{INTRODUCTION}

Photosynthesis begins with the absorption of a photon which creates an excited state on a pigment molecule. The excitation is transferred between the pigments of lightharvesting complexes until it arrives at a reaction center (RC) in the pigment-protein complex, where the photon energy is used for the later dark stages of charge separation and conversion of energy from physical into chemical one. The efficiency of the energy transfer is very high, with a near unity yield [1]. This sparks the long-standing and increasing interest in the understanding of the physics behind the energy conversion within photosynthesis [2]. Recently, much attention has been paid to the role of quantum coherence: growing experimental evidence [3-9] and theoretical models [10-22] reveal that quantum coherence contributes beneficially to the high efficiency. Understanding the underlying mechanism of such natural system can assist us in designing novel nanofabricated structures for quantum transport and optimized solar cells.

Viewing the photosynthetic reaction center as a quantum heat engine (QHE), the authors of Ref.[23] analyzed the charge separation in light-harvesting complexes. This treatment bridges the two seemingly unrelated effects attributed to quantum coherence in natural(photosynthesis) and artificial(photovoltaics) light-harvesting systems. The common ground between photovoltaics and photosynthesis has also been investigated recently in [24, 25]. In analogy with a continuous Carnot-like cycle, Dorfman et al. showed that the power of a photocell based on Photosystem II reaction center (PSII RC) can be increased by $27 \%$ attributed to noiseinduced quantum coherence-Fano interference [26, 27],

*Corresponding address: yixx@nenu.edu.cn which was found in artificial photocells and lasing without inversion. Creatore et al. proposed the other mechanism and showed that the dipole-dipole interaction between two neighboring electron donors play a key role in enhancing the current and power of the photocell [28]. They claimed that this increasing can be up to $35 \%$.

In these studies, only one charge-separation pathway is considered. Identifying the primary electron donors and dominating charge-separation pathways has been a question of recent extensive research and debate. At the moment, there is much evidence that two main pathways make significant contribution under ambient condition [29-32]. This motivates us to ask the question that whether a multi-pathway scheme is more beneficial to designing artificial light-harvesting devices? In this paper, we will answer this question via numerical simulation for the current and power as well as the robustness of the current and power against the charge recombination and dephasing.

The remainder of the paper is organized as follows. In Sec. II, we introduce a model of a quantum heat engine inspired by PSII RC to describe the charge separation, which includes a second pathway in comparison with the model proposed in [23, 28]. A master equation describing the evolution of the two-pathway system is also derived in this section. In Sec. III, the concepts of effective voltage $V$ and power are introduced and used to characterize the charge separation, new results due to the second pathway are discussed. In Sec. IV, we focus on how the multiple pathways affect the behavior of $j-V$ and $P-V$ features. We explain the advantage of multiple pathways over simply increasing the decay rates and cross-couplings. In Sec. V, we take the charge recombination and dephasing that might coexist in natural PSII RC into consideration. Numerical results of one- and multi-pathway models are shown, from which we further see the advantage of multiple pathways. Sec. VI is devoted to concluding remarks. 


\section{MODEL}

To present the model, we first illustrate the structure of the Photosystem II reaction center complex in Fig.1. The six pigment molecules are closely spaced in particular positions and orientations, and coupled by the dipole-dipole interactions resulting in exciton states. These pigments are distributed in two branches of protein matrix: $D 1$ and $D 2$. $P_{D 1}$ and $P_{D 2}$, a special pair of coupled chlorophylls, is located at the center of the PSII RC. They contribute mostly to the lowest energy states and are the primary electron donors, forming two exciton states which are denoted as $\left|a_{1}\right\rangle$ and $\left|a_{2}\right\rangle$. These two pairs of molecules are also coupled to the accessory chlorophylls $A c c_{D 1}$ and $A c c_{D 2}$ located in the two different branches $D 1$ and $D 2$, respectively. $P h e_{D 1}$ and $P h e_{D 2}$ are the remaining two pheophytin pigments coupled to the rest of the molecules, and act as the electron acceptors. Photosynthetic antennae transfer energy of the absorbed solar photons to the RC where the transmembrane charge separation takes place. Charge separation in the core of pigment-protein $\mathrm{RC}$ complexes is the first energy conversion step in photosynthesis. The consequent electrochemical potential drives a chain of chemical reactions, including the reduction of NADP to NADPH, the synthesis of ATP and the oxidized part of the RC splits water, releasing molecular oxygen, all these leading eventually to the stable storage of solar energy.

The photosynthetic reaction center may be analyzed as a biological quantum heat engine(BQHE) that transforms highenergy thermal photon radiation into low-entropy electron flux. As shown in Fig. 2 (a), a five-level QHE scheme describes the photoinduced charge separation between the donor and the acceptor molecules interacting with thermal light. State $|b\rangle$ represents the lowest energy configuration in which both donors and acceptor molecules are in the ground state. States $\left|a_{1}\right\rangle$ and $\left|a_{2}\right\rangle$ correspond to single-exciton states in the first and second donors, respectively. $|\alpha\rangle$ is the chargeseparated state with the excited electron transferred from the donors to the acceptor molecules. And $|\beta\rangle$ describes the ionized state in which the system is positively charged (the excited electron is assumed to have been used to perform work). The heat engine cycle begins with the excited electron promoted from $|b\rangle$ to $\left|a_{1}\right\rangle$ and/or $\left|a_{2}\right\rangle$ after the absorption of a solar photon. This excited electron is then transferred to the acceptor by emission of a phonon (transition from $\left|a_{1}\right\rangle$ and/or $\left|a_{2}\right\rangle$ to $|\alpha\rangle$, corresponding to the formation of a radical pair $P_{D 2}^{+} A c c_{D 2}^{-}$in Fig.11). Furthermore, the electron can be released from state $|\alpha\rangle$ with a rate $\Gamma$, resulting in a current from $|\alpha\rangle$ to $|\beta\rangle$ driving a chain of chemical reactions, leading eventually to the stable storage of solar energy. The current $j=e \Gamma \rho_{\alpha \alpha}$ is thus determined by the relaxation rate $\Gamma$ and the population of $|\alpha\rangle$. The acceptor-to-donor charge recombination represented by the decay rate $\Gamma_{\alpha \rightarrow b}=\chi \Gamma$, with $\chi$ a dimensionless fraction, brings the system back to the ground state $|b\rangle$ but does not produce current, limiting the power output of our QHE [28, 33]. Finally, to complete the cycle, we allow another population transfer to take place, emitting a phonon with excess energy, bringing the electron back to the neutral ground state $|b\rangle$ with a rate $\Gamma_{c}$. We also assume that the donor ex- cited states $\left|a_{1}\right\rangle$ and $\left|a_{2}\right\rangle$ undergo a dephasing process (via a rate $\left.\Gamma_{d e p}\right)$, which will influence the current and power generated. This model including one charge-separation pathway $(|\alpha\rangle \rightarrow|\beta\rangle)$ can exhibit noise-induced quantum coherence due to Fano interference, which originates from the coupling of two levels to a common reservoir and thus the effect can be revealed by the cross-couplings. Fano interference can minimize the acceptor-to-donor charge recombination by inducing coherence between $\left|a_{1}\right\rangle$ and $\left|a_{2}\right\rangle$. This brings about enhanced current and power output as we will show in section II.

Although electron transfer in the PSII RC has been thoroughly investigated and several charge-separation pathways that involve the formation of several different radical pairs were identified, works on the effect of multiple chargeseparation pathways are rarely found. At the moment, much evidence shows that two main pathways make significant contributions under ambient conditions [29-32]. Therefore we emphatically study two pathways, i.e., we add another acceptor molecule that consists of a charged-separated state and an ionized state to the model in Fig. 2 (a). As shown in Fig. 2 (b), the initial excitation of states $\left|a_{1}\right\rangle$ and $\left|a_{2}\right\rangle$ can be transferred to the two acceptor molecules in states $\left|\alpha_{1}\right\rangle$ and $\left|\alpha_{2}\right\rangle$, respectively, with the excess energy radiated as a phonon, and further produce electric current and returning back to $|b\rangle$ via $\left|\beta_{1}\right\rangle$ and/or $\left|\beta_{2}\right\rangle$. Here, the total current in the two pathways is given by $j=e \Gamma_{1} \rho_{\alpha_{1} \alpha_{1}}+e \Gamma_{2} \rho_{\alpha_{2} \alpha_{2}}$. The structures of RCs from green plants, algae, and bacteria differ in the nature and precise orientation of the constituent pigments, resulting in different spectroscopic and dynamical parameters. Nevertheless, our six-level scheme may be applied to all RCs as discussed below.

With this knowledge, the dynamics of our heat engine can be described by the following master equation,

$$
\frac{d \hat{\rho}_{S}}{d t}=-i\left[H_{S}, \hat{\rho}_{S}\right]+L \hat{\rho}_{S}
$$

where $H_{S}=\sum_{\substack{j=b, a_{1}, a_{2}, \alpha_{1}, \alpha_{2}, \beta_{1}, \beta_{2}}} E_{j}|j\rangle\langle j|$ is the system Hamiltonian, with $E_{j}$ the energy of the $i$ th level. The superoperator $L$ describing the effect of reservoirs and acceptor-donor recombinations can be decomposed as

$$
\begin{aligned}
L \hat{\rho}_{S}= & L_{h} \hat{\rho}_{S}+L_{c 1} \hat{\rho}_{S}+L_{c 2} \hat{\rho}_{S}+L_{r e l} \hat{\rho}_{S} \\
& +L_{d e p} \hat{\rho}_{S}+L_{r e c} \hat{\rho}_{S} .
\end{aligned}
$$

For the high temperature photon reservoir (HTR), its effects can be described by

$$
\begin{aligned}
L_{h} \hat{\rho}_{S}= & \sum_{k, l=1,2} \frac{\gamma_{k l h}}{2}\left[( n _ { h } + 1 ) \left(\sigma_{h l} \hat{\rho}_{S} \sigma_{h k}^{\dagger}+\sigma_{h k} \hat{\rho}_{S} \sigma_{h l}^{\dagger}\right.\right. \\
& \left.-\sigma_{h l}^{\dagger} \sigma_{h k} \hat{\rho}_{S}-\hat{\rho}_{S} \sigma_{h k}^{\dagger} \sigma_{h l}\right)+n_{h}\left(\sigma_{h k}^{\dagger} \hat{\rho}_{S} \sigma_{h l}\right. \\
& \left.\left.+\sigma_{h l}^{\dagger} \hat{\rho}_{S} \sigma_{h k}-\sigma_{h k} \sigma_{h l}^{\dagger} \hat{\rho}_{S}-\hat{\rho}_{S} \sigma_{h l} \sigma_{h k}^{\dagger}\right)\right],
\end{aligned}
$$

where $\hat{\sigma}_{h k}=|b\rangle\left\langle a_{k}\right|(k=1,2)$, and $n_{h}$ is the average number of solar photons. $\gamma_{k l h}(k=l)$ denotes the decay rate, where $\gamma_{11 h}=\gamma_{1 h}$ and $\gamma_{22 h}=\gamma_{2 h}$ represents the spontaneous decay from the upper level $\left|a_{1}\right\rangle$ and $\left|a_{2}\right\rangle$ to level $|b\rangle$, respectively. 


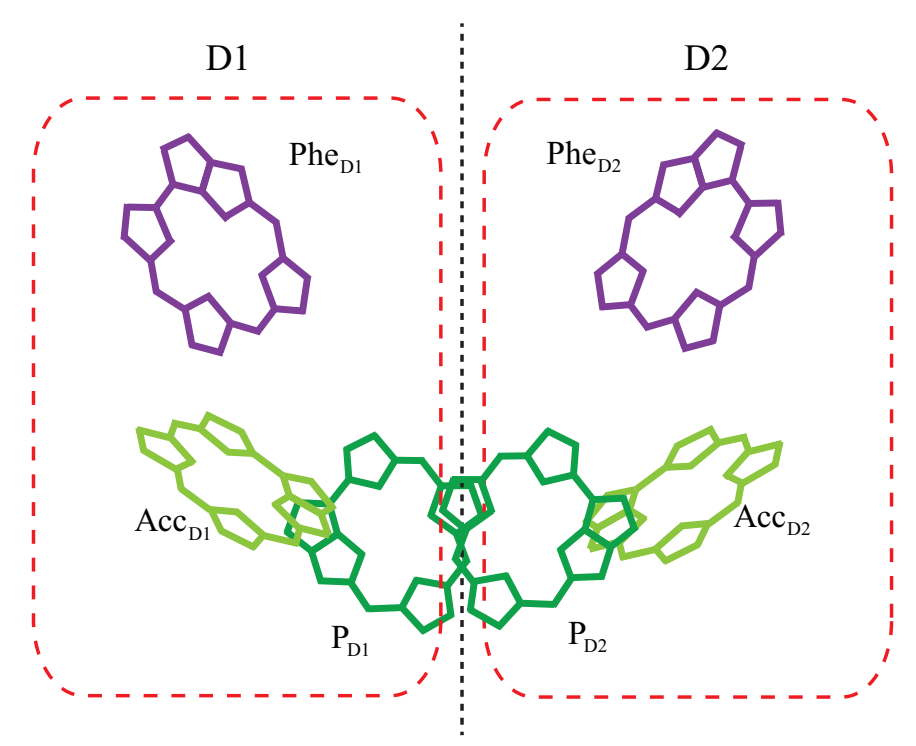

FIG. 1: (Color online) Arrangement of six core-pigments in the PSII RC. It consists of the special pair $\mathrm{P}_{\mathrm{D} 1}$, $\mathrm{P}_{\mathrm{D} 2}$, the two accessory chlorophylls $\mathrm{Acc}_{\mathrm{D} 1}, \mathrm{Acc}_{\mathrm{D} 2}$, and the two pheophytins Phe $\mathrm{D} 1, \mathrm{Phe}_{\mathrm{D} 2}$.

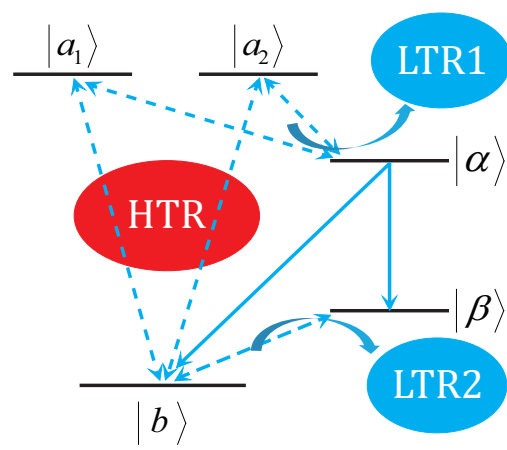

(a)

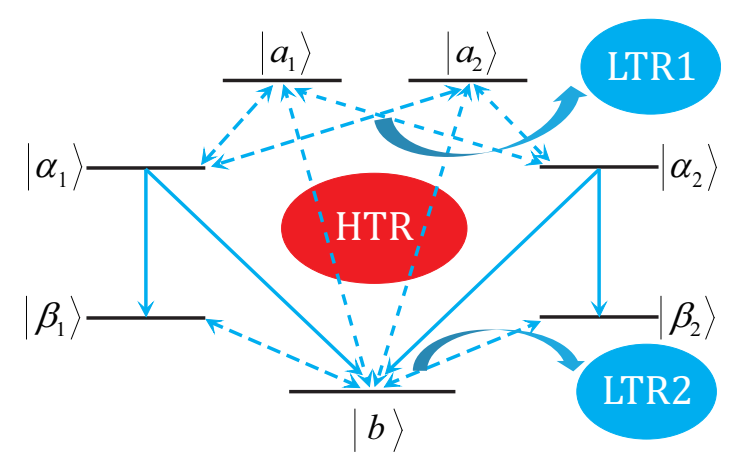

(b)

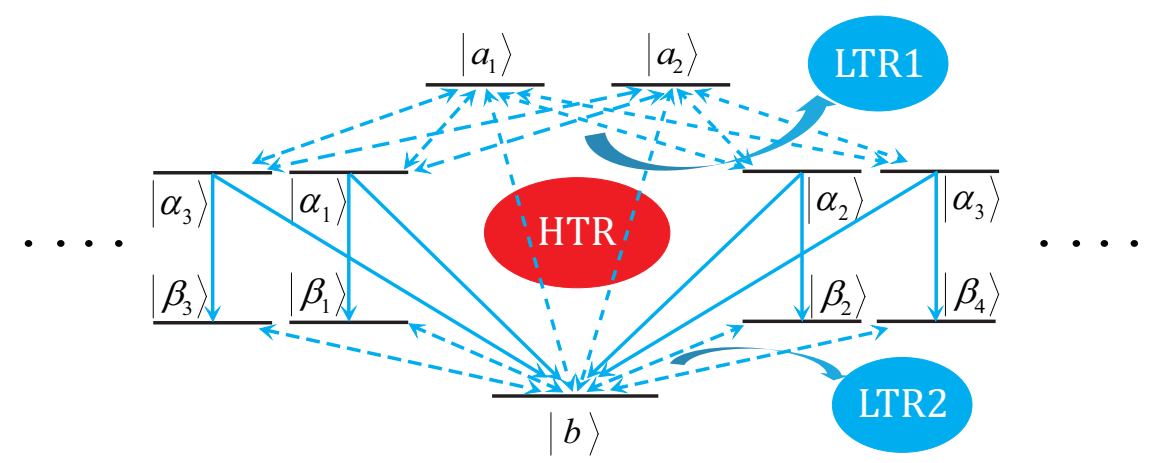

(c)

FIG. 2: (Color online) Schemes of the BQHE model based on the photosynthetic reaction center. HTR denotes the high-temperature photon reservoir, while LTR1 and LTR2 stand for the low-temperature phonon reservoirs. HTR induces transition from the ground state $|b\rangle$ to the single-exciton states $\left|a_{1}\right\rangle$ and/or $\left|a_{2}\right\rangle$. LTR1 induces transition from $\left|a_{1}\right\rangle$ and/or $\left|a_{2}\right\rangle$ to the charge-separated state $|\alpha\rangle$ ( $\left|\alpha_{1}\right\rangle$ and $\left|\alpha_{2}\right\rangle$ for (b), $\left|\alpha_{i}\right\rangle$ $(i=1,2,3 \ldots)$ for (c)). LTR2 induces transition from the ionized state $|\beta\rangle\left(\left|\beta_{1}\right\rangle\right.$ and $\left|\beta_{2}\right\rangle$ for $(\mathrm{b}),\left|\beta_{i}\right\rangle(i=1,2,3 \ldots)$ for (c)) to the ground state. The three models differ in the number of donor, i.e., pathway. 
The noise-induced quantum coherence is closely related to the terms with $\gamma_{k l h}(k \neq l)$, which we will refer to as crosscouplings. They describe the effect of interference, and we will assume $\gamma_{12 h}=\gamma_{21 h}$, and $\gamma_{k l h}=\eta_{h} \sqrt{\gamma_{k h} \gamma_{l h}}(k \neq l)$ where $\eta_{h}=1$ represents the maximal coherence and $\eta_{h}=0$ the minimal coherence. It is worth noting that $\eta_{h}=0$ represents a case in which the two donor molecules interact with their own (independent) reservoirs. Thus there is no coherence induced by noise [34] in this situation.

Superoperator $L_{c 2}$ corresponding to the second low temperature phonon reservoir (LTR2) has the form

$$
\begin{aligned}
L_{c 2} \hat{\rho}_{S}= & \sum_{k, l=1,2} \frac{\Gamma_{k l c}}{2}\left[( n _ { c 2 } + 1 ) \left(\sigma_{c l} \hat{\rho}_{S} \sigma_{c k}^{\dagger}+\sigma_{c k} \hat{\rho}_{S} \sigma_{c l}^{\dagger}\right.\right. \\
& \left.-\sigma_{c l}^{\dagger} \sigma_{c k} \hat{\rho}_{S}-\hat{\rho}_{S} \sigma_{c k}^{\dagger} \sigma_{c l}\right)+n_{c 2}\left(\sigma_{c k}^{\dagger} \hat{\rho}_{S} \sigma_{c l}\right. \\
& \left.\left.+\sigma_{c l}^{\dagger} \hat{\rho}_{S} \sigma_{c k}-\sigma_{c k} \sigma_{c l}^{\dagger} \hat{\rho}_{S}-\hat{\rho}_{S} \sigma_{c l} \sigma_{c k}^{\dagger}\right)\right],
\end{aligned}
$$

where $\hat{\sigma}_{c k}=|b\rangle\left\langle\beta_{k}\right|(k=1,2)$ denotes the lower operator. $n_{c 2}$ is the average phonon number of the cold (phonon) reservoir LTR2. $\Gamma_{k l c}(k=l)$ is the corresponding decay rate, with $\Gamma_{11 c}=\Gamma_{1 c}$ and $\Gamma_{22 c}=\Gamma_{2 c}$ representing the spontaneous decay from the upper level $\left|\beta_{1}\right\rangle$ and $\left|\beta_{2}\right\rangle$ to level $|b\rangle$, respectively; and $\Gamma_{k l c}(k \neq l)$ is a cross-coupling that describes the effect of interference, with $\Gamma_{12 c}=\Gamma_{21 c}$. In numerical simulations, we will set $\Gamma_{k l c}=\eta_{c_{2}} \sqrt{\Gamma_{k c} \Gamma_{l c}}(k \neq l)$ with $\eta_{c_{2}}=1$ representing the maximal coherence and $\eta_{c_{2}}=0$ the minimal coherence.

The quantum coherence induced by the low temperature phonon reservoir (LTR1) can be described by,

$$
\begin{aligned}
L_{c 1} \hat{\rho}_{S}= & \sum_{k, l, m, n=1,2} \frac{\gamma_{k l m n}}{2}\left[( n _ { c 1 } + 1 ) \left(\sigma_{k l} \hat{\rho}_{S} \sigma_{m n}^{\dagger}\right.\right. \\
& \left.+\sigma_{m n} \hat{\rho}_{S} \sigma_{k l}^{\dagger}-\sigma_{k l}^{\dagger} \sigma_{m n} \hat{\rho}_{S}-\hat{\rho}_{S} \sigma_{m n}^{\dagger} \sigma_{k l}\right) \\
& +n_{c 1}\left(\sigma_{m n}^{\dagger} \hat{\rho}_{S} \sigma_{k l}+\sigma_{k l}^{\dagger} \hat{\rho}_{S} \sigma_{m n}-\sigma_{m n} \sigma_{k l}^{\dagger} \hat{\rho}_{S}\right. \\
& \left.\left.-\hat{\rho}_{S} \sigma_{k l} \sigma_{m n}^{\dagger}\right)\right]
\end{aligned}
$$

where $\hat{\sigma}_{k l}=\left|\alpha_{k}\right\rangle\left\langle a_{l}\right|(k, l=1,2)$ or $\hat{\sigma}_{m n}=\left|\alpha_{m}\right\rangle\left\langle a_{n}\right|(m, n=$ $1,2)$, and $n_{c 1}$ is the average phonon numbers of LTR1. $\gamma_{k l m n}(k l=m n)$ are decay rates from the upper levels $\left|a_{1}\right\rangle$ and $\left|a_{2}\right\rangle$ to the lower levels $\left|\alpha_{1}\right\rangle$ and $\left|\alpha_{2}\right\rangle$, respectively. For $k l \neq m n$, terms with $\gamma_{k l m n}$ would induce quantum coherence leading to the interference. Note that the cross-couplings here are complicated more than those of HTR or LTR2. There are two types of cross-couplings due LTR1. One is the same as that in previous works [23, 34], defined as $\gamma_{k l m n}=\eta_{c_{1}}^{1} \sqrt{\gamma_{k l} \gamma_{m n}}$ $(k l \neq m n)$, including $\gamma_{1121}$ and $\gamma_{1222}$. These two crosscouplings represented by terms with $\gamma_{12 c}$ in the model shown in Fig. 2(a). The other one is defined as $\gamma_{k l m n}=\eta_{c_{1}}^{2} \sqrt{\gamma_{k l} \gamma_{m n}}$ $(k l \neq m n)$, including terms with $\gamma_{1112}, \gamma_{1122}, \gamma_{1221}, \gamma_{2122}$. These terms are not considered in the model depicted in Fig.2 (a). The first type of cross-couplings couple $\left|a_{1}\right\rangle$ and $\left|a_{2}\right\rangle$ with the same lower lever. Namely, terms with $\gamma_{1121}$ couple $\left|a_{1}\right\rangle$ and $\left|a_{2}\right\rangle$ to $\left|\alpha_{1}\right\rangle$, while terms with $\gamma_{1222}$ to $\left|\alpha_{2}\right\rangle$. In contrast, the second type of cross-couplings, the lower levels are different. In the later numerical simulations, we will choose $\eta_{c_{1}}^{1,2}=1$ representing the maximal coherence and 0 the minimal coherence. We will discuss the effects caused by these cross-couplings in section IV.
$L_{r e l}$ describes a process that the system in state $\left|\alpha_{1}\right\rangle\left(\left|\alpha_{2}\right\rangle\right)$ decays to state $\left|\beta_{1}\right\rangle\left(\left|\beta_{2}\right\rangle\right)$. It leads to the electronic current proportional to the relaxation rates $\Gamma_{1}$ and $\Gamma_{2}$ as defined later.

$$
\begin{aligned}
L_{r e l} \hat{\rho}_{S}= & \sum_{k=1,2} \frac{\Gamma_{k}}{2}\left(\left|\beta_{k}\right\rangle\left\langle\alpha_{k}\left|\hat{\rho}_{S}\right| \alpha_{k}\right\rangle\left\langle\beta_{k}\right|\right. \\
& \left.-\left|\alpha_{k}\right\rangle\left\langle\alpha_{k}\left|\hat{\rho}_{S}-\hat{\rho}_{S}\right| \alpha_{k}\right\rangle\left\langle\alpha_{k}\right|\right) .
\end{aligned}
$$

We describe the dephasing of the system on states $\left|a_{1}\right\rangle$ and $\left|a_{2}\right\rangle$ by

$$
\begin{aligned}
L_{d e p} \hat{\rho}_{S}= & \sum_{k=1,2} \frac{\Gamma_{d e p}}{2}\left(\left|a_{k}\right\rangle\left\langle a_{k}\left|\hat{\rho}_{S}\right| a_{k}\right\rangle\left\langle a_{k}\right|\right. \\
& \left.-\left|a_{k}\right\rangle\left\langle a_{k}\left|\hat{\rho}_{S}-\hat{\rho}_{S}\right| a_{k}\right\rangle\left\langle a_{k}\right|\right) .
\end{aligned}
$$

with $\Gamma_{\text {dep }}$ being the dephasing rate.

Finally, the loss channel due to acceptor-to-donor charge recombination is described by,

$$
\begin{aligned}
L_{r e c} \hat{\rho}_{S}= & \sum_{k=1,2} \frac{\chi \Gamma_{k}}{2}\left(|b\rangle\left\langle\alpha_{k}\left|\hat{\rho}_{S}\right| \alpha_{k}\right\rangle\langle b|\right. \\
& \left.-\left|\alpha_{k}\right\rangle\left\langle\alpha_{k}\left|\hat{\rho}_{S}-\hat{\rho}_{S}\right| \alpha_{k}\right\rangle\left\langle\alpha_{k}\right|\right) .
\end{aligned}
$$

Here the dimensionless $\chi$ stands for the recombination rate.

\section{EFFECTS OF CROSS-COUPLINGS}

TABLE I: Parameters used in the numerical simulations.

\begin{tabular}{clc}
\hline \hline Parameters & Units & Values \\
\hline$E_{a_{i} b}$ & $\mathrm{~cm}^{-1}$ & 14,856 \\
$E_{\beta_{i} b}=E_{a_{i} \alpha_{j}}$ & $\mathrm{~cm}^{-1}$ & 1,611 \\
$\gamma_{i h}$ & $\mathrm{~cm}^{-1}$ & 0.01 \\
$\gamma_{i i i i}\left(=\gamma_{2 c}\right)$ & $\mathrm{cm}^{-1}$ & 140 \\
$\gamma_{i j i j(i \neq j)}\left(=\gamma_{1 c}\right)$ & $\mathrm{cm}^{-1}$ & 18 \\
$\Gamma_{i c}\left(=\Gamma_{c}\right)$ & $\mathrm{cm}^{-1}$ & 200 \\
$\Gamma_{i}(=\Gamma)$ & $\mathrm{cm}^{-1}$ & 1,000 \\
$\Gamma_{d e p}$ & $\mathrm{~cm}^{-1}$ & 41 \\
$n_{h}$ & & 60,000 \\
$n_{c}$ & & $4.4 \times 10^{-4}$ \\
$N_{c}$ & & $4.4 \times 10^{-4}$ \\
$\chi$ & & 0.2 \\
\hline \hline
\end{tabular}


In the present system, the current generated can be thought to flow across a load connecting the acceptor levels $|\alpha\rangle$ and $|\beta\rangle$. Introducing an effective voltage $V$ as a drop of the electrostatic potential across the load, we obtain $e V=E_{\alpha}-E_{\beta}+k_{B} T \ln \left(\frac{\hat{\rho}_{\alpha \alpha}}{\hat{\rho}_{\beta \beta}}\right)$ for the model in Fig. 2 (a), where $e$ is the electric charge and $E_{i}$ is the energy of state $|i\rangle$.

In our model shown in Fig. 2] (b), choosing $E_{i_{1}}=E_{i_{2}}$ ( $i=a, \alpha, \beta)$ and setting all parameters the same for the two pathways, we have $e V=E_{\alpha_{1}}-E_{\beta_{1}}+k_{B} T \ln \left(\frac{\rho_{\alpha_{1} \alpha_{1}}}{\rho_{\beta_{1} \beta_{1}}}\right)$, and $e V=E_{\alpha_{2}}-E_{\beta_{2}}+k_{B} T \ln \left(\frac{\rho_{\alpha_{2} \alpha_{2}}}{\rho_{\beta_{2} \beta_{2}}}\right)$, which define the voltage $V$. The current, accordingly, is defined by $j=e \Gamma_{1} \rho_{\alpha_{1} \alpha_{1}}+e \Gamma_{2} \rho_{\alpha_{2} \alpha_{2}}$. It is similar to the parallel circuit in classical electromagnetism. Based on the current and the voltage, we easily obtain the power output $P=j \cdot V$. We apply this to the steady-state of the system to characterize the performance of our BQHE and the photovoltaic properties of the complex, i.e., the steady-state current-voltage $(j-V)$ and power-voltage $(P-V)$ characteristics. Using the steady-state solution of Eq. (1), we plot the $j-V$ curve and power with different $\Gamma$, while keep the other parameters fixed. Note that $\Gamma \rightarrow 0(j \rightarrow 0)$ corresponds to the open-circuit case, and in the short-circuit case, $V \rightarrow 0$.

The parameters used in our simulation are listed in Table I. These parameters are chosen from recent literature, e.g., [23, 35- 37] and they are used in the simulation in [23, 28, 34]. The energy differences are defined as $E_{a_{i} b}=E_{a_{i}}-E_{b}, E_{\beta_{i} b}=$ $E_{\beta_{i}}-E_{b}$ and $E_{a_{i} \alpha_{j}}=E_{a_{i}}-E_{\alpha_{j}}$, where $i, j=1,2$. Parameters in the brackets of Table I are used in the one-pathway model in Fig. 2 (a). In the following, we will discuss the effect of cross-couplings separately.

\section{A. Cross-coupling $\Gamma_{12 c}$ induced by LTR2}

Interestingly, the $j-V$ and $P-V$ behaviors demonstrate that as cross-coupling strength $\Gamma_{12 c}$ increases, the current and power decrease, see Fig. 3 (e) and (f). This is different from the results in previous works [23, 34]. Note that, the parameters chosen for the two pathways are the same (see, Table I), thus the population on $\left|a_{1}\right\rangle$ and $\left|a_{2}\right\rangle$ is the same, so is the population on $\left|\alpha_{1}\right\rangle$ and $\left|\alpha_{2}\right\rangle,\left|\beta_{1}\right\rangle$ and $\left|\beta_{2}\right\rangle$. From Fig. 3](d), we see that, the maximal $\Gamma_{12 c}\left(\eta_{c_{2}}=1\right)$ benefits the population on $\left|\beta_{1}\right\rangle$ and $\left|\beta_{2}\right\rangle$ and leads this population dominant over the others. Therefore we cannot plot $j-V$ characteristics in this case as $\rho_{\alpha_{1} \alpha_{1}}=\rho_{\alpha_{2} \alpha_{2}}=0$. It was shown in Ref. [23, 34] that the power is increased and the current-voltage characteristic of the heat engine gets better with Fano interference. Here Fano interference characterized by $\Gamma_{12 c}$, however, shows an opposite effect. We will explain this observation in subsection D. So, in the following numerical simulations, we will take $\Gamma_{12 c}=0$.

\section{B. Cross-couplings induced by LTR1}

Fig. 4 shows that, current and power are enhanced as $\gamma_{11,21}$ and $\gamma_{12,22}$ increase, which coincides with the results predicted
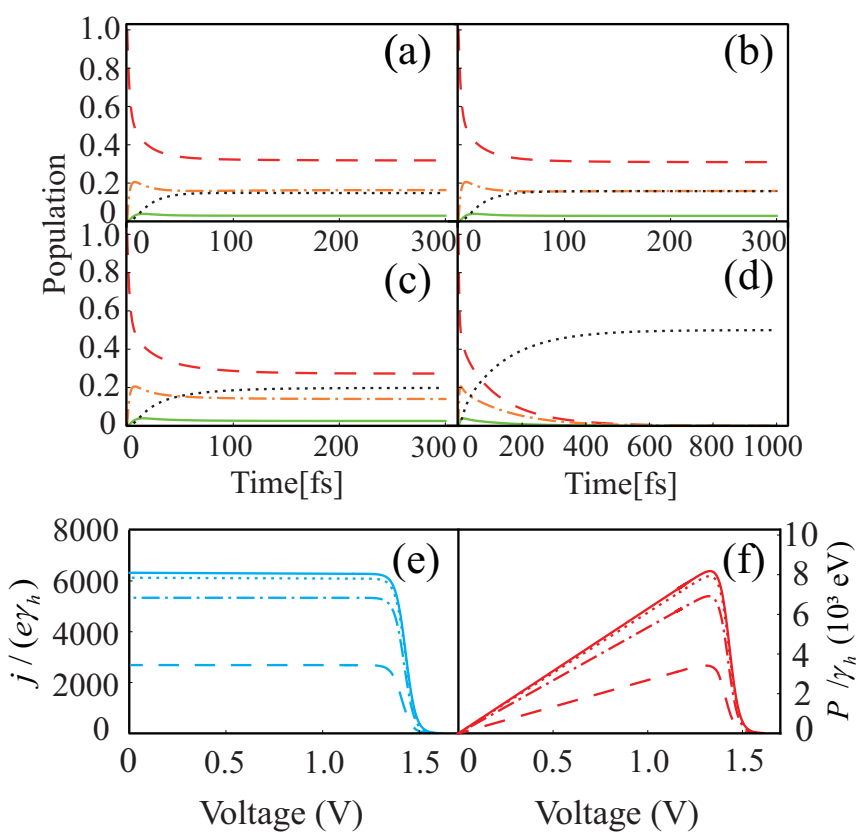

FIG. 3: (Color online) Fig. 3 (a)-(d) show the time evolution of the population on each level of two-pathway model corresponding to Fig. 2 (b) at room temperature $T=300 \mathrm{~K}: \rho_{b b}$ in red-dashed, $\rho_{a_{1} a_{1}}$ $\left(\rho_{a_{2} a_{2}}\right)$ in orange-dot-dashed, $\rho_{\alpha_{1} \alpha_{1}}\left(\rho_{\alpha_{2} \alpha_{2}}\right)$ in green-solid, and $\rho_{\beta_{1} \beta_{1}}$ $\left(\rho_{\beta_{2} \beta_{2}}\right)$ in black-dotted line. The cross-coupling $\Gamma_{12 c}=\eta_{c_{2}} \sqrt{\Gamma_{1 c} \Gamma_{2 c}}$ takes $\eta_{c_{2}}=0,0.3,0.6,1$ for (a), (b), (c) and (d), respectively. The other cross-couplings $\gamma_{12 h}=\eta_{h} \sqrt{\gamma_{1 h} \gamma_{2 h}}, \gamma_{k l m n}=\eta_{c_{1}}^{1} \sqrt{\gamma_{k l} \gamma_{m n}}(k l \neq$ $m n)$ and $\gamma_{k l m n}=\eta_{c_{1}}^{2} \sqrt{\gamma_{k l} \gamma_{m n}}(k l \neq m n)$ take the maximal values, i.e., $\eta_{h}=\eta_{c_{1}}^{1}=\eta_{c_{1}}^{2}=1$. (e) and (f) are plotted for the current and power generated as a function of effective voltage $V$ at room temperature: $\eta_{c_{2}}=0$ in solid, $\eta_{c_{2}}=0.3$ in dotted, $\eta_{c_{2}}=0.6$ in dot-dashed and $\eta_{c_{2}}=0.9$ in dashed line.

by Dorfman et al. This gives rise to a question that why does $\Gamma_{12 c}$ exhibit effects different from that by $\gamma_{11,21}$ and $\gamma_{12,22}$ ?

Fig. 5 shows that, population in each state seems no difference for any values of the second type of cross-couplings $\gamma_{k l m n}=\eta_{c_{1}}^{2} \sqrt{\gamma_{k l} \gamma_{m n}}(k l \neq m n)$. Thus different from couplings with $\gamma_{1121}\left(\gamma_{1222}\right)$ and $\gamma_{12 c}$, the second type of cross-couplings has marginal effects on the current and power. This is analogous to quantum beats, which can occur in a $V$ type atom, but can not in a $\Lambda$ type atom based on the theory of quantum electrodynamics (QED) [38], as shown in Fig.6] $V$-type atom in excited states decays via the emission of a photon with frequency $v_{1}$ or $v_{2}$. Since both emissions lead to the same final state, we cannot determine along which path, $v_{1}$ or $v_{2}$, the atom decays. This uncertainty leads to an interference between $v_{1}$ and $v_{2}$, exhibiting quantum beats. A $\Lambda$-type atom also decays via the emission of a photon with frequency $v_{1}$ or $v_{2}$. However, an observation of atom at a long time after the emission can tell us which channel (1 or 2) was taken (atom in $\left|b_{1}\right\rangle$ or $\left|b_{2}\right\rangle$ ). Consequently, we expect no beats in this case. For the model of Fig. 2 (b), transitions described by the second type of cross-couplings lead to different lower states, which do not induce interference. Thus the second type of cross-couplings has no effect on the $j-V$ and $P-V$ behav- 

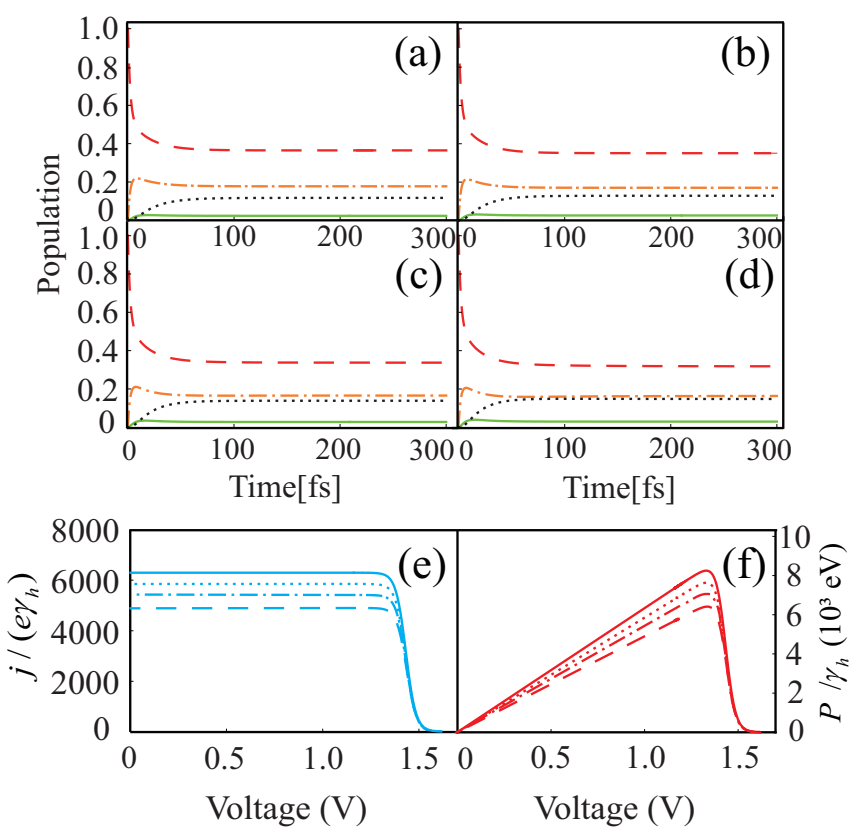

FIG. 4: (Color online) Fig. 4 (a) - (d) show the time evolution of the population on each level in the two-pathway model shown in Fig. 2 (b) at room temperature $T=300 \mathrm{~K} . \rho_{b b}$ was plotted in red-dashed, $\rho_{a_{1} a_{1}}\left(\rho_{a_{2} a_{2}}\right)$ in orange-dot-dashed, $\rho_{\alpha_{1} \alpha_{1}}\left(\rho_{\alpha_{2} \alpha_{2}}\right)$ in green-solid, and $\rho_{\beta_{1} \beta_{1}}\left(\rho_{\beta_{2} \beta_{2}}\right)$ in black-dotted line. $\eta_{c_{1}}^{1}$ in the cross-couplings $\gamma_{k l m n}=$ $\eta_{c_{1}}^{1} \sqrt{\gamma_{k l} \gamma_{m n}}(k l \neq m n)$ takes $\eta_{c_{1}}^{1}=0,0.3,0.6,1$ for (a), (b), (c) and (d), respectively. The other cross-couplings $\gamma_{12 h}=\eta_{h} \sqrt{\gamma_{1 h} \gamma_{2 h}}$ and $\gamma_{k l m n}=\eta_{c_{1}}^{2} \sqrt{\gamma_{k l} \gamma_{m n}}(k l \neq m n)$ take the maximal values, i.e., $\eta_{h}=$ $\eta_{c_{1}}^{2}=1$. As mentioned in the last subsection, $\Gamma_{12 c}=0$. (e) and (f) are plotted for the current and power generated as a function of effective voltage $V$ at room temperature. Dashed line is for $\eta_{c_{1}}^{1}=0$, dot-dashed for $\eta_{c_{1}}^{1}=0.3$, dotted line for $\eta_{c_{1}}^{1}=0.6$, and $\eta_{c_{1}}^{1}=1$ is ploted in thin-solid line.

iors.

With this consideration, in the following numerical simulations, we take $\gamma_{k l m n}=\eta_{c_{1}}^{2} \sqrt{\gamma_{k l} \gamma_{m n}}=0$.

\section{Cross-couplings induced by HTR}

As expected, $\gamma_{12 h}$ benefits the transition as seen from Fig.7 Inspired by the physics behind the quantum beat, we claim that interference can play an important role in the current and power only when the upper two levels $\left|a_{1}\right\rangle$ and $\left|a_{2}\right\rangle$ are coherently excited at the initial time. When the coherence between $\left|a_{1}\right\rangle$ and $\left|a_{2}\right\rangle$ is absent, i.e., seting $\gamma_{12 h}=0, \gamma_{12 c}$ itself may suppress the exciton transfer. Equivalently, setting $\gamma_{12 c}=0, \gamma_{12 h}$ itself may inhibit the current and power. In terms of steadystate solution to Eq. 1, we further confirm this observation, and it shows how these two types of interference work together to affect the transitions.
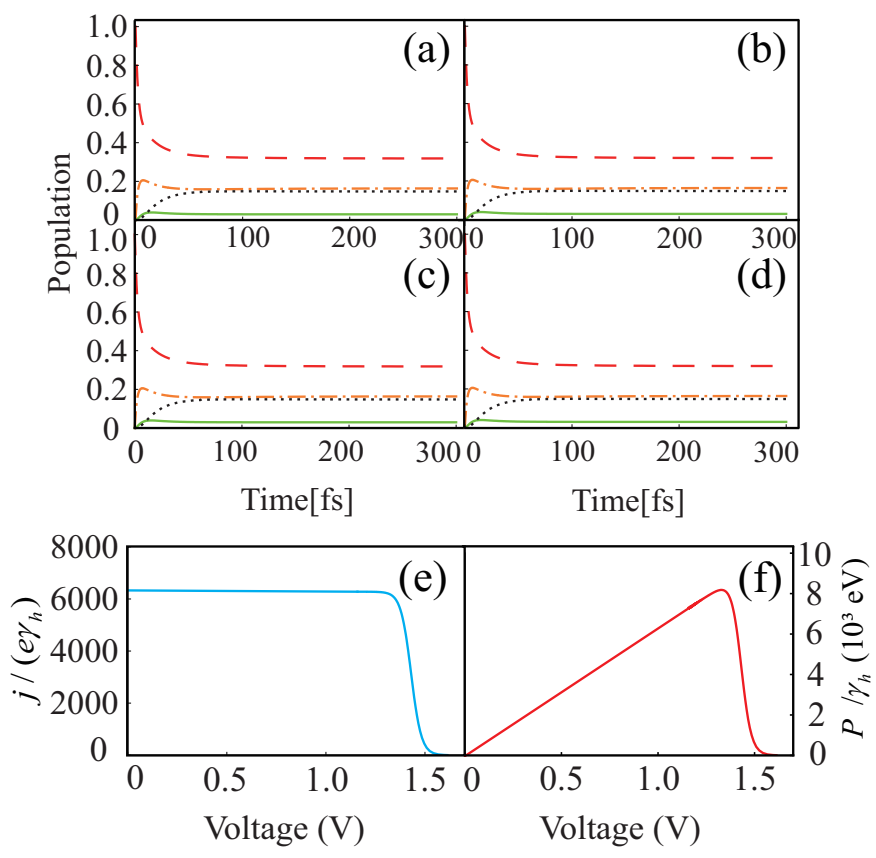

FIG. 5: (Color online) Fig. 5.(a)-(d) show the time evolution of the population on each level of the system depicted in Fig. 2(b) at room temperature $T=300 \mathrm{~K}: \rho_{b b}$ in red-dashed, $\rho_{a_{1} a_{1}}\left(\rho_{a_{2} a_{2}}\right)$ in orangedot-dashed, $\rho_{\alpha_{1} \alpha_{1}}\left(\rho_{\alpha_{2} \alpha_{2}}\right)$ in green-solid, and $\rho_{\beta_{1} \beta_{1}}\left(\rho_{\beta_{2} \beta_{2}}\right)$ in blackdotted line. The cross-couplings $\gamma_{k l m n}=\eta_{c_{1}}^{2} \sqrt{\gamma_{k l} \gamma_{m n}}(k l \neq m n)$ take $\eta_{c_{1}}^{2}=0,0.3,0.6,1$ for (a), (b), (c) and (d), respectively. The other cross-couplings $\gamma_{12 h}=\eta_{h} \sqrt{\gamma_{1 h} \gamma_{2 h}}$ and $\gamma_{k l m n}=\eta_{c_{1}}^{1} \sqrt{\gamma_{k l} \gamma_{m n}}(k l \neq m n)$ take the maximal values, i.e., $\eta_{h}=\eta_{c_{1}}^{1}=1$. $\Gamma_{12 c}=0$. (e) and (f) are plotted for the current and power as a function of effective voltage $V$ at room temperature $T=300 \mathrm{~K}$. Actually, we plot four curves in (e) and (f) with $\eta_{c_{1}}^{2}=0,0.3,0.6,1$, respectively, although all them overlap. Since (a)-(d) do not show any difference for different $\eta_{c_{1}}^{2}$, the $j-V$ and $P-V$ behaviors are exactly the same for different $\eta_{c_{1}}^{2}$.

\section{Combined effects of HTR and LTR1}

For simplicity, we plot the steady-state $j-V$ characteristic and power for one-pathway model in Fig. 8. We set $\gamma_{12 c}=\eta_{c_{1}}^{\prime} \sqrt{\gamma_{1 c} \gamma_{2 c}}$ and $\gamma_{12 h}=\eta_{h} \sqrt{\gamma_{1 h} \gamma_{2 h}}$, thus $\eta_{c_{1}}^{\prime}=1$ $\left(\eta_{h}=1\right)$ corresponds to the maximal coherence and $\eta_{c_{1}}^{\prime}=0$ $\left(\eta_{h}=0\right)$ the minimal coherence. We observe that, when $\gamma_{12 h}$ is very small, the current and power decrease with the increasing of $\gamma_{12 c}$. Similarly, when $\gamma_{12 c}$ is very small, large $\gamma_{12 h}$ inhibits the transitions. Recall the quantum beats, if one of $\gamma_{12 h}$ and $\gamma_{12 c}$ is very small, $\left|a_{1}\right\rangle$ and $\left|a_{2}\right\rangle$ are almost independently excited. For this reason, when one cross-coupling term becomes very large, the current and power will be suppressed. When both $\gamma_{12 h}$ and $\gamma_{12 c}$ increase to a certain value, strong crosscouplings can enhance the current and power. This conclusion holds for $\gamma_{1121}$ and $\gamma_{1222}$ in the two-pathway model. For the terms with $\Gamma_{12 c}$ discussed in subsection $\mathrm{A},\left|\beta_{1}\right\rangle$ and $\left|\beta_{2}\right\rangle$ obviously, are not in a coherent state. As a result, the transition process is suppressed as $\Gamma_{12 c}$ increases. 


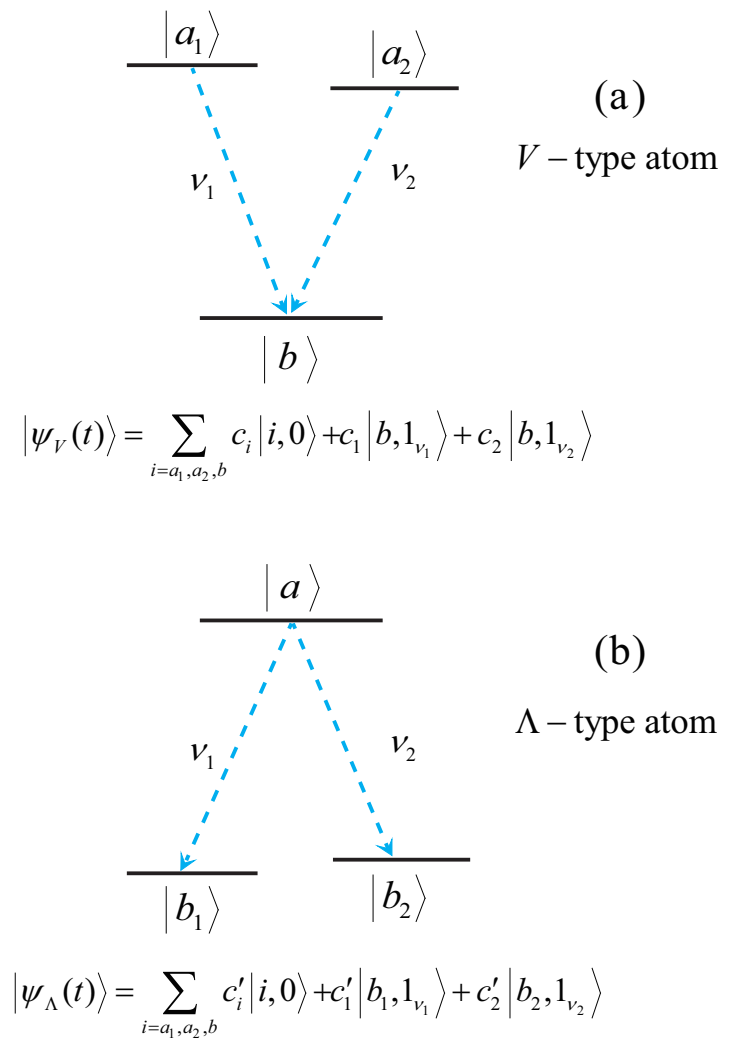

FIG. 6: (Color online) Three-level atomic structures for (a) $V$-type and (b) $\Lambda$-type quantum beat.

\section{EFFECT OF MULTIPLE PATHWAYS}

Electron transfer in the PSII RC has been thoroughly studied and several charge-separation pathways were identified. In this section, we investigate the role of multiple pathways on the behavior of $j-V$ and $P-V$. The simulation results are presented in Fig. 9 From Fig. 9 we find that as the number of pathways increases, the BQHE produces stronger current and power. Especially, the model of two pathways, corresponding to Fig. 2 (b), shows a current enhancement of $76.8 \%$, comparing with the case of only one pathway shown in Fig. 2 (a). Nevertheless, with the number of pathways increase further, the enhancement rate decreases. The current of three-pathway model shows $25.9 \%$ more than that of two-pathway model, and the current of four-pathway model just $16.7 \%$ more than that of three-pathway one. The observations can be understood as follows: the current and power of the BQHE sharply depends on the rate of transition from the ground state $|b\rangle$ to state $\left|a_{1}\right\rangle$ or $\left|a_{2}\right\rangle$, i.e., the decay rate in HTR. More pathways mathematically equal to increasing the strength of the decay rate in LTR1 and LTR2. When $\gamma_{1 h}, \gamma_{2 h}$ and $\gamma_{12 h}$ take a fixed value, the energy flux density is fixed. The strength of the decay rate in LTR1 and LTR2, or rather the number of pathways should match the transport rate in HTR, otherwise, excessive pathways can not significantly improve the transition process. We further propose that in order to accelerate the transfer process, multiple pathways in the BQHE (or similar structure in
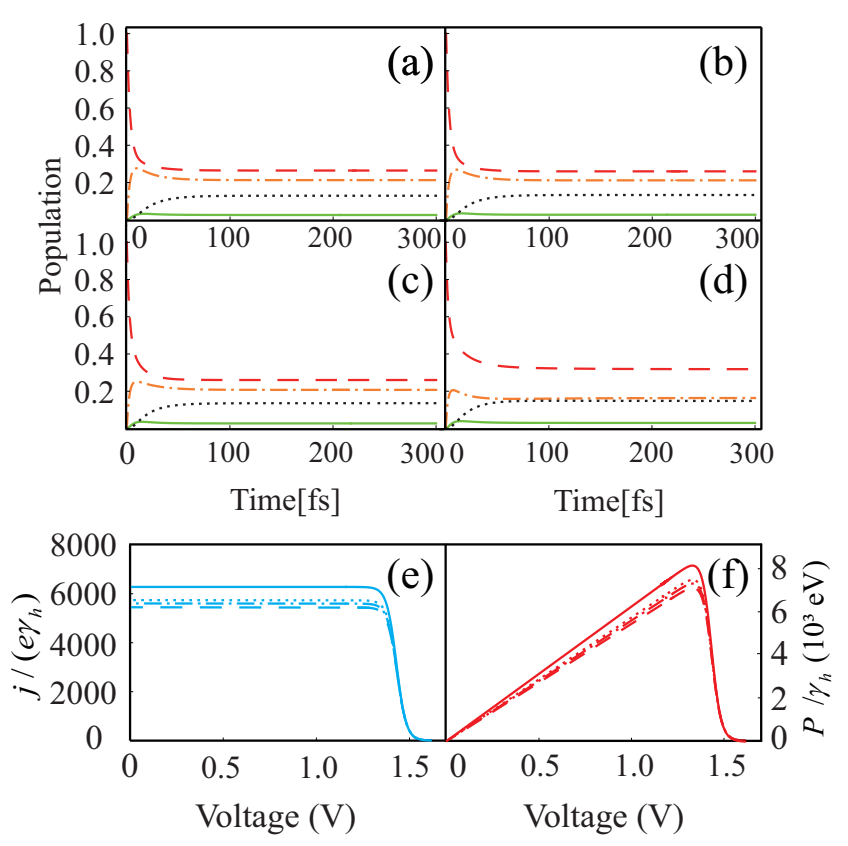

FIG. 7: (Color online) Fig. 7 (a)-(d) show the time evolution of the population on each level of the system in Fig. 2 (b) at room temperature $T=300 \mathrm{~K} . \rho_{b b}$ is shown in red-dashed, $\rho_{a_{1} a_{1}}\left(\rho_{a_{2} a_{2}}\right)$ in orange-dot-dashed, $\rho_{\alpha_{1} \alpha_{1}}\left(\rho_{\alpha_{2} \alpha_{2}}\right)$ in green-solid, and $\rho_{\beta_{1} \beta_{1}}\left(\rho_{\beta_{2} \beta_{2}}\right)$ in black-dotted line. The cross-couplings $\gamma_{12 h}=\eta_{h} \sqrt{\gamma_{1 h} \gamma_{2 h}}$ take $\eta_{h}=0,0.3,0.6,1$ for (a), (b), (c) and (d), respectively. The other cross-couplings $\gamma_{k l m n}=\eta_{c_{1}}^{1} \sqrt{\gamma_{k l} \gamma_{m n}}(k l \neq m n)$ takes the maximal value, i.e., $\eta_{c_{1}}^{1}=1 . \gamma_{k l m n}=\eta_{c_{1}}^{2} \sqrt{\gamma_{k l} \gamma_{m n}}=0(k l \neq m n)$ and $\Gamma_{12 c}=0$. (e) and (f) are plotted for the current and power as a function of effective voltage $V$ at room temperature. $\eta_{h}=0$ is plotted in dashed, $\eta_{h}=0.3$ in dot-dashed, $\eta_{h}=0.6$ in dotted and $\eta_{h}=1$ in thin-solid line.

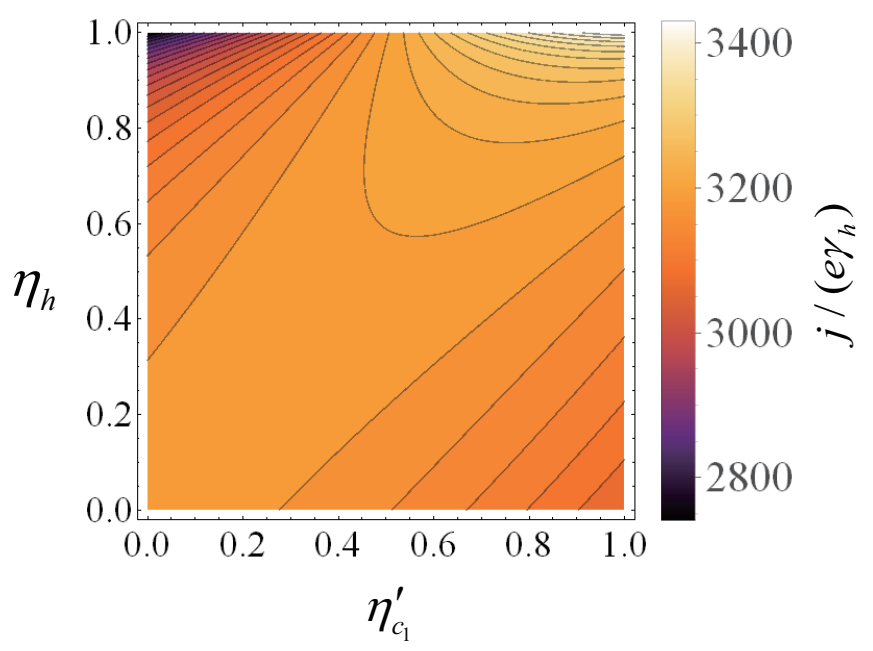

FIG. 8: (Color online) Counter plot of the current as a function of cross-couplings $\gamma_{12 c}$ and $\gamma_{12 h}$. As $\gamma_{12 c}=\eta_{c_{1}}^{\prime} \sqrt{\gamma_{1 c} \gamma_{2 c}}\left(\gamma_{12 h}=\right.$ $\left.\eta_{h} \sqrt{\gamma_{1 h} \gamma_{2 h}}\right), \eta_{c_{1}}^{\prime}=1\left(\eta_{\mathrm{h}}=1\right)$ corresponds to the maximal coherence and $\eta_{c_{1}}^{\prime}=0\left(\eta_{\mathrm{h}}=0\right)$ the minimal coherence. 


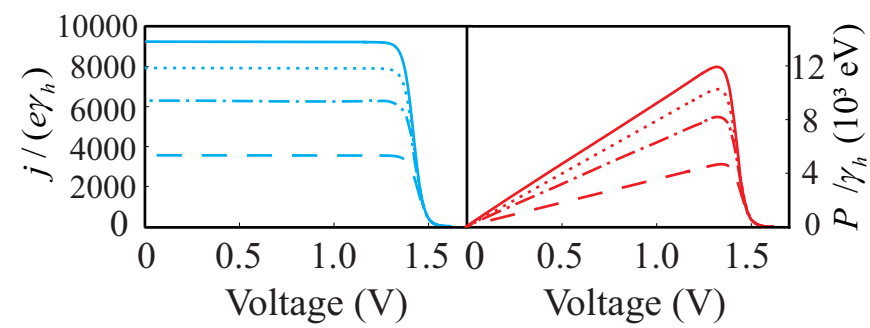

FIG. 9: (Color online) The current and power as a function of effective voltage $V$ at room temperature for different numbers of pathway. The results for one pathway are shown in dashed, two pathways in dot-dashed, three in dotted, four in thin-solid line. Cross-couplings corresponding to LTR2 and the second type of cross-couplings corresponding to LTR1 are set to 0 . All the other cross-couplings take their maximal values.

the natural photosynthetic reaction center) are a better choice comparing with simply increasing the strength of the decay rates and cross-couplings related to LTR1 and LTR2 within one-pathway model. As shown in Appendix, the decay rates or cross-couplings are given by $\gamma_{k l m n}=\frac{2 p^{2} V_{p h n} g_{k l p} g_{m n p}}{\pi c}$, from which we know that these rates are determined by the ambient phonon reservoir and the diploe moment of $\left|a_{1,2}\right\rangle \leftrightarrow|b\rangle$ for LTR1 $\left(\left|\beta_{1,2}\right\rangle \leftrightarrow|b\rangle\right.$ for LTR2). The natural condition of reservoir is hot and wet, and generally unstable. And the structure of RC complexes is born fixed, resulting in constant diploe moment between different states of pigment molecules. Therefore, these factors cannot be utilized to enhance the reaction efficiency. Nevertheless, more charge-separation pathways means increasing reaction channels. This seems easy to achieve. In the next section, considering acceptor-to-donor charge recombination and noise-induced dephasing, we will show that multiple pathways is still a better choice in contrast with increasing the strength of the decay rate in LTR1 and LTR2.

\section{EFFECTS OF RECOMBINATION AND DEPHASING}

In this section, we examine how charge recombination at the acceptor/donor interface and dephasing of $\left|a_{1}\right\rangle$ and $\left|a_{2}\right\rangle$ affects the current and power. In Fig. 10 (a), we plot the relative change of current as a function of the recombination rate $\chi$. The relative change of current is defined by $(j(\chi=0)-j(\chi)) / j(\chi=0)$. We calculate this relative change for both cases of maximal coherence and minimal coherence with one and two pathways, respectively. To determine which one, multiple pathways or large decay rates in LTR1 and LTR2, is a better choice, we double these decay rates and the relaxation rate in one-pathway model with the other parameters fixed. Fig. 10 (b) shows the relative current change for both cases of maximal coherence and minimal coherence with one and three pathways, respectively. Similarly, we triple these decay rates and the relaxation rate in one-pathway model with the other parameters fixed. Fig. 10 (a) and Fig. 10 (b) show that as the recombination rate increases, the current
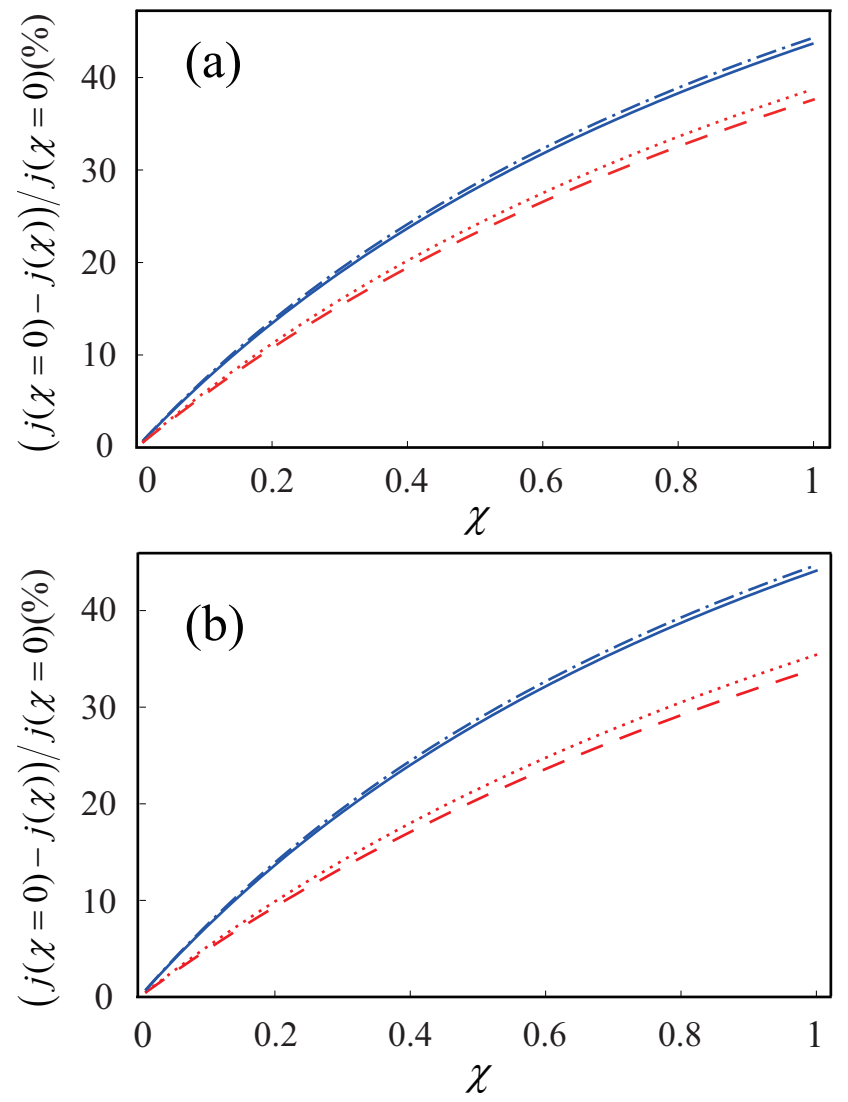

FIG. 10: (Color online) (a) is plotted for the current ratio defined by $(j(\chi=0)-j(\chi)) / j(\chi=0)$ as a function of the recombination rate for one- and two-pathway models. The blue-solid and red-dashed lines are for the maximal coherence, i.e., all the cross-couplings take the maximal values, except that cross-couplings induced by LTR 2 and the second type of cross-couplings induced by LTR 1 are set to 0 . The blue-dot-dashed and red-dotted lines are for the minimal coherence, i.e., all the cross-couplings take the minimal values 0 . The blue lines stand for one-pathway model with the strength of the decay rates in LTR1 and LTR2 and the relaxation rate doubled and the other parameters fixed. The red lines stand for two-pathway one. (b) is the case of one- and three-pathway models. The blue-solid and red-dashed lines are for the maximal coherence while the blue-dot-dashed and red-dotted lines are for the minimal coherence. The blue lines stand for one-pathway model with the strength of the decay rates in LTR1 and LTR2 and the relaxation rate tripled and the other parameters fixed. The red lines stand for three-pathway one.

decreases monotonically as expected. Nevertheless, the setup with maximal coherence can better reduce the impact of acceptor-to-donor charge recombination. Therefore, noiseinduced coherence between $\left|a_{1}\right\rangle$ and $\left|a_{2}\right\rangle$ due to Fano interference can diminish the influence of recombination. Besides, we observe that the larger number of the pathways is, the more robust the system against the recombination. Therefore, in the presence of the additional loss mechanism due to acceptor-to-donor charge recombination, the maximal coherence scheme/multi-pathway set-up is more efficient than the minimal coherence scheme/one-pathway one.

Next we explore whether, in the presence of dephasing, the 
multi-pathway set-up can still benefit the current and power. We calculate the relative change of current as a function of the dephasing rate $\Gamma_{d e p}$ and present the results in Fig.11 The relative change of current has also been calculated for different numbers of pathways. Similarly, to compare the two choices of multiple pathways and increasing the strength of the decay rate in LTR1 and LTR2, we modify these decay rates and the relaxation rate in one-pathway model with the other parameters fixed, just as in the case of the relaxation rate discussed above. Observing the results, we find that the current decreases monotonically as the dephasing rate increases. Besides, the more pathways, the more robust against the dephasing. Therefore, the multiple-pathway model is more efficient than the one-pathway one even in the presence of depasing process mechanism.
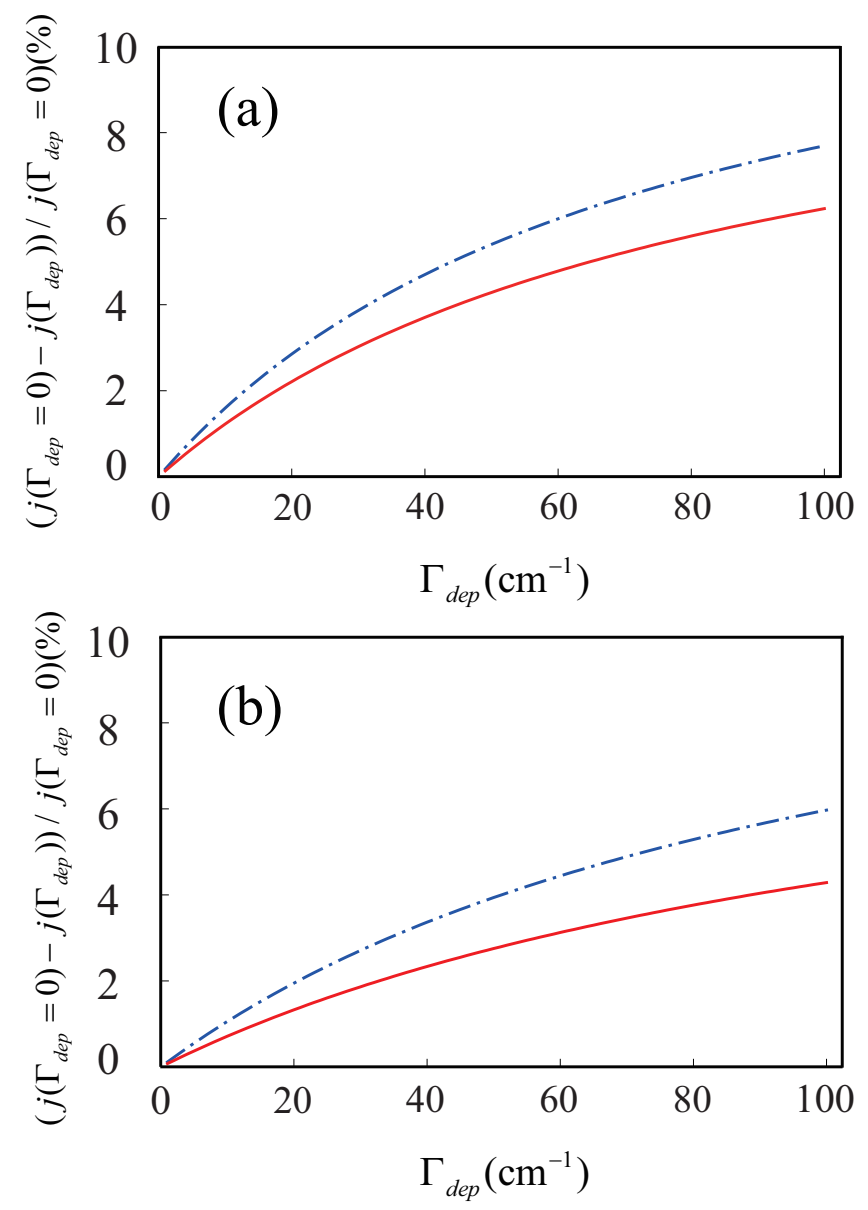

FIG. 11: (Color online) (a) is the relative current change (defined by $\left.\left(j\left(\Gamma_{\text {dep }}=0\right)-j\left(\Gamma_{d e p}\right)\right) / j\left(\Gamma_{d e p}=0\right)\right)$ as a function of dephasing rate. The blue-dashed line is for one-pathway model with the strength of the decay rates in LTR1 and LTR2 and the relaxation rate doubled and the other parameters fixed. The red-solid line is for two-pathway one. (b) is the case of one- and three-pathway model. The bluedashed line is for one-pathway model with the strength of the decay rates in LTR1 and LTR2 and the relaxation rate tripled and the other parameters fixed. The red-solid line is for three-pathway one. The dimensionless parameter $\chi=0.2$ as in Table I.

\section{CONCLUSION}

In conclusion, we have studied the dynamics of twopathway biological quantum heat engine and calculated the steady-state current-voltage $(j-V)$ and power-voltage $(P-V)$ characteristics. We explored the effect of various crosscouplings, and found that only the cross-couplings describing transitions between high levels and the same lower energy level have effects on the $j-V$ and $P-V$ behaviors. We explained the physics behind this observation using the concept of quantum beat. Furthermore we find that the current and power can be increased due to Fano interference when the upper two levels are in a coherent supposition. Finally, we calculate the $j-V$ and $P-V$ characteristics subjected to acceptorto-donor charge recombination and dephasing, and show that noise-induced quantum coherence helps in the suppression of the influence of the acceptor-to-donor charge recombination. These results suggest that multi-pathway BQHE can benefit the charge separation and the light-harvesting yields, and it is a better choice in contrast with increasing the strength of decay rates and cross-couplings.

\section{ACKNOWLEDGMENTS}

This work is supported by National Natural Science Foundation of China (NSFC) under Grants No. 11175032, and No. 61475033 .

\section{Appendix A: The derivation of Eq. (1)}

In this part, we will present a derivation for the master equation used in the maintext. We consider a total Hamiltonian

$$
\hat{H}=\hat{H}_{S}+\hat{H}_{R}+\hat{H}_{S R}
$$

where the system Hamiltonian

$$
\hat{H}_{S}=\hbar \omega_{b}|b\rangle\left\langle b\left|+\hbar \omega_{a_{1}}\right| a_{1}\right\rangle\left\langle a_{1}\left|+\hbar \omega_{a_{2}}\right| a_{2}\right\rangle\left\langle a_{2}\right|,
$$

with $\hbar \omega_{a_{i}, b}=E_{a_{i}, b}(i=1,2)$ being the energy of states $\left|a_{i}\right\rangle$, $|b\rangle$.

$\hat{H}_{R}$ represents the Hamiltonian of the high temperature photon reservoir (HTR)

$$
\hat{H}_{R}=\sum_{p} \hbar v_{p} \hat{b}_{p}^{\dagger} \hat{b}_{p}
$$

Here $\hat{b}_{p}^{\dagger}$ and $\hat{b}_{p}$ denote the creation and destruction operators. The interaction Hamiltonian in the rotating-wave approximation (RWA) is

$$
\hat{H}_{S R}=\hbar \sum_{p} g_{1 p} \hat{\sigma}_{h 1} \hat{b}_{p}^{\dagger}+\hbar \sum_{q} g_{2 q} \hat{\sigma}_{h 2} \hat{b}_{q}^{\dagger}+\text { H.c. }
$$

with $\hat{\sigma}_{h 1}=|b\rangle\left\langle a_{1}\right|$ and $\hat{\sigma}_{h 2}=|b\rangle\left\langle a_{2}\right|$ being the lower operators. The coupling constant $g_{1 p}=-\frac{\wp_{a_{1}}, \hat{\epsilon}_{p} \mathscr{E}_{p}}{\hbar}$ and $g_{2 q}=$ $-\frac{\wp_{a_{2} b} \hat{\epsilon}_{\epsilon_{G}} \mathscr{E}_{p}}{\hbar}$, where $\wp_{a_{1} b}\left(\wp_{a_{2} b}\right)$ is the dipole moment of $\left|a_{1}\right\rangle \leftrightarrow$ 
$|b\rangle\left(\left|a_{2}\right\rangle \leftrightarrow|b\rangle\right) . \hat{\epsilon}_{p}\left(\hat{\epsilon}_{q}\right)$ is the polarization of the field. The Substituting $\hat{V}(t)$ into Eq. (A6), we have, electric field per photon is $\mathscr{E}_{p, q}=\left(\frac{\hbar v_{p, q}}{2 \epsilon_{p} V}\right)^{\frac{1}{2}}$.

It is convenient to work in the interaction picture. The Hamiltonian, in the interaction picture, is given by

$$
\begin{aligned}
\hat{V}(t)= & \hbar \sum_{k} g_{1 p} \hat{b}_{p}^{\dagger} \hat{\sigma}_{h 1} e^{-i\left(\omega_{a_{1} b}-v_{p}\right) t} \\
& +\hbar \sum_{q} g_{2 q} \hat{b}_{q}^{\dagger} \hat{\sigma}_{h 2} e^{-i\left(\omega_{a_{2} b}-v_{q}\right) t}+\text { H.c.. }
\end{aligned}
$$

The equation of motion for the system density operator $\hat{\rho}_{S}$ is

$$
\begin{aligned}
\frac{d \hat{\rho}_{S}(t)}{d t}= & -\frac{i}{\hbar} \operatorname{Tr}_{R}\left[\hat{V}(t), \hat{\rho}_{S}(0) \otimes \hat{\rho}_{R}(0)\right] \\
& -\frac{1}{\hbar^{2}} \operatorname{Tr}_{R} \int_{0}^{t}\left[\hat{V}(t),\left[\hat{V}\left(t^{\prime}\right), \hat{\rho}_{S}\left(t^{\prime}\right) \otimes \hat{\rho}_{R}(0)\right]\right] d t^{\prime} .
\end{aligned}
$$

$$
\begin{aligned}
& \frac{d \hat{\rho}_{S}(t)}{d t}=-\int_{0}^{t} d t^{\prime}\left\{\sum_{p, p^{\prime}} g_{1 p} g_{1 p^{\prime}} e^{i\left(\omega_{a_{1} b}-v_{p}\right) t-i\left(\omega_{a_{1} b}-v_{p^{\prime}}\right) t^{\prime}} \operatorname{Tr}_{R}\left[\hat{\sigma}_{h 1}^{+} \hat{b}_{p},\left[\hat{b}_{p^{\prime}}^{\dagger} \hat{\sigma}_{h 1}, \hat{\rho}_{S}\left(t^{\prime}\right) \otimes \hat{\rho}_{R}(0)\right]\right]\right. \\
& +\sum_{p, q^{\prime}} g_{1 p} g_{2 q^{\prime}} e^{i\left(\omega_{a_{1} b}-v_{p}\right) t-i\left(\omega_{a_{2} b}-v_{q^{\prime}}\right) t^{\prime}} \operatorname{Tr}_{R}\left[\hat{\sigma}_{h 1}^{+} \hat{b}_{p},\left[\hat{b}_{q^{\prime}}^{\dagger} \hat{\sigma}_{h 2}, \hat{\rho}_{S}\left(t^{\prime}\right) \otimes \hat{\rho}_{R}(0)\right]\right] \\
& +\sum_{q, p^{\prime}} g_{2 q} g_{1 p^{\prime}} e^{i\left(\omega_{a_{2} b}-v_{q}\right) t-i\left(\omega_{a_{1} b}-v_{p^{\prime}}\right) t^{\prime}} \operatorname{Tr}_{R}\left[\hat{\sigma}_{h 2}^{+} \hat{b}_{q},\left[\hat{b}_{p^{\prime}}^{\dagger} \hat{\sigma}_{h 1}, \hat{\rho}_{S}\left(t^{\prime}\right) \otimes \hat{\rho}_{R}(0)\right]\right] \\
& +\sum_{q, q^{\prime}} g_{2 q} g_{2 q^{\prime}} e^{i\left(\omega_{a_{2} b}-v_{q}\right) t-i\left(\omega_{a_{2} b} b v_{q^{\prime}}\right) t^{\prime}} \operatorname{Tr}_{R}\left[\hat{\sigma}_{h 2}^{+} \hat{b}_{q},\left[\hat{b}_{q^{\prime}}^{\dagger} \hat{\sigma}_{h 2}, \hat{\rho}_{S}\left(t^{\prime}\right) \otimes \hat{\rho}_{R}(0)\right]\right] \\
& +\sum_{p, p^{\prime}} g_{1 p} g_{1 p^{\prime}} e^{-i\left(\omega_{a_{1} b}-v_{p}\right) t+i\left(\omega_{a_{1} b}-v_{p^{\prime}}\right) t^{\prime}} \operatorname{Tr}_{R}\left[\hat{b}_{p}^{\dagger} \hat{\sigma}_{h 1},\left[\hat{\sigma}_{h 1}^{+} \hat{b}_{p^{\prime}}, \hat{\rho}_{S}\left(t^{\prime}\right) \otimes \hat{\rho}_{R}(0)\right]\right] \\
& +\sum_{p, q^{\prime}} g_{1 p} g_{2 q^{\prime}} e^{-i\left(\omega_{a_{1} b}-v_{p}\right) t+i\left(\omega_{a_{2} b}-v_{q^{\prime}}\right) t^{\prime}} \operatorname{Tr}_{R}\left[\hat{b}_{p}^{\dagger} \hat{\sigma}_{h 1},\left[\hat{\sigma}_{h 2}^{+} \hat{b}_{q^{\prime}}, \hat{\rho}_{S}\left(t^{\prime}\right) \otimes \hat{\rho}_{R}(0)\right]\right] \\
& +\sum_{q, p^{\prime}} g_{2 q} g_{1 p^{\prime}} e^{-i\left(\omega_{a_{2} b}-v_{q}\right) t+i\left(\omega_{a_{1} b}-v_{p^{\prime}}\right) t^{\prime}} \operatorname{Tr}_{R}\left[\hat{b}_{q}^{\dagger} \hat{\sigma}_{h 2},\left[\hat{\sigma}_{h 1}^{+} \hat{b}_{p^{\prime}}, \hat{\rho}_{S}\left(t^{\prime}\right) \otimes \hat{\rho}_{R}(0)\right]\right] \\
& \left.+\sum_{q, q^{\prime}} g_{2 q} g_{2 q^{\prime}} e^{-i\left(\omega_{a_{2} b}-v_{q}\right) t+i\left(\omega_{\left.a_{2} b^{-}-v_{q^{\prime}}\right) t^{\prime}}\right.} \operatorname{Tr}_{R}\left[\hat{b}_{q}^{\dagger} \hat{\sigma}_{h 2},\left[\hat{\sigma}_{h 2}^{+} \hat{b}_{q^{\prime}}, \hat{\rho}_{S}\left(t^{\prime}\right) \otimes \hat{\rho}_{R}(0)\right]\right]\right\} .
\end{aligned}
$$

The sum over $p$ may be replaced by an integral through

$$
\sum_{p} \rightarrow \frac{V_{p h t}}{\pi^{2}} \int_{0}^{\infty} d p p^{2}
$$

where $V_{p h t}$ is the volume. Neglecting all memory effects and assuming that the density matrix is a slowly varying function of time, i.e., $\hat{\rho}\left(t^{\prime}\right) \approx \hat{\rho}(t)$, we obtain the integration over time as

$$
\int_{0}^{\infty} d t^{\prime} e^{i\left(\omega-v_{k}\right)\left(t-t^{\prime}\right)}=\pi \delta\left(\omega-v_{k}\right)
$$

Substitute A8 and A9 into A7p and note that $\left\langle\hat{b}_{p}\right\rangle=$ $\left\langle\hat{b}_{p}^{\dagger}\right\rangle=0,\left\langle\hat{b}_{p} \hat{b}_{p^{\prime}}\right\rangle=\left\langle\hat{b}_{p}^{\dagger} \hat{b}_{p^{\prime}}^{\dagger}\right\rangle=0,\left\langle\hat{b}_{p}^{\dagger} \hat{b}_{p^{\prime}}\right\rangle=\bar{n}_{p} \delta_{p p^{\prime}}$, and $\left\langle\hat{b}_{p} \hat{b}_{p^{\prime}}^{\dagger}\right\rangle=\left(\bar{n}_{p}+1\right) \delta_{p p^{\prime}}$. The master equation reduces to 


$$
\begin{aligned}
\frac{d \hat{\rho}_{S}(t)}{d t}= & \frac{V_{p h t}}{\pi c}\left\{p^{2} g_{1 p}^{2}\left[\left(\bar{n}_{p}+1\right)\left(2 \hat{\sigma}_{h 1} \hat{\rho}_{S}(t) \hat{\sigma}_{h 1}^{\dagger}-\hat{\sigma}_{h 1}^{\dagger} \hat{\sigma}_{h 1} \hat{\rho}_{S}(t)-\hat{\rho}_{S}(t) \hat{\sigma}_{h 1}^{\dagger} \hat{\sigma}_{h 1}\right)\right]\right. \\
& +p^{2} g_{1 p}^{2}\left[\bar{n}_{p}\left(2 \hat{\sigma}_{h 1}^{\dagger} \hat{\rho}_{S}(t) \hat{\sigma}_{h 1}-\hat{\sigma}_{h 1} \hat{\sigma}_{h 1}^{\dagger} \hat{\rho}_{S}(t)-\hat{\rho}_{S}(t) \hat{\sigma}_{h 1} \hat{\sigma}_{h 1}^{\dagger}\right)\right] \\
& +p^{2} g_{2 p}^{2}\left[\left(\bar{n}_{p}+1\right)\left(2 \hat{\sigma}_{h 2} \hat{\rho}_{S}(t) \hat{\sigma}_{h 2}^{\dagger}-\hat{\sigma}_{h 2}^{\dagger} \hat{\sigma}_{h 2} \hat{\rho}_{S}(t)-\hat{\rho}_{S}(t) \hat{\sigma}_{h 2}^{\dagger} \hat{\sigma}_{h 2}\right)\right] \\
& +p^{2} g_{2 p}^{2}\left[\bar{n}_{p}\left(2 \hat{\sigma}_{h 2}^{\dagger} \hat{\rho}_{S}(t) \hat{\sigma}_{h 2}-\hat{\sigma}_{h 2} \hat{\sigma}_{h 2}^{\dagger} \hat{\rho}_{S}(t)-\hat{\rho}_{S}(t) \hat{\sigma}_{h 2} \hat{\sigma}_{h 2}^{\dagger}\right)\right] \\
& +p^{2} g_{1 p} g_{2 p}\left[\left(\bar{n}_{p}+1\right)\left(\hat{\sigma}_{h 1} \hat{\rho}_{S}(t) \hat{\sigma}_{h 2}^{\dagger}+\hat{\sigma}_{h 2} \hat{\rho}_{S}(t) \hat{\sigma}_{h 1}^{\dagger}\right)\right] \\
& +p^{2} g_{1 p} g_{2 p}\left[\bar{n}_{p}\left(\hat{\sigma}_{h 1}^{\dagger} \hat{\rho}_{S}(t) \hat{\sigma}_{h 2}+\hat{\sigma}_{h 2}^{\dagger} \hat{\rho}_{S}(t) \hat{\sigma}_{h 1}\right)\right] \\
& +p^{2} g_{1 p} g_{2 p}\left[\left(\bar{n}_{p}+1\right)\left(\hat{\sigma}_{h 2} \hat{\rho}_{S}(t) \hat{\sigma}_{h 1}^{\dagger}+\hat{\sigma}_{h 1} \hat{\rho}_{S}(t) \hat{\sigma}_{h 2}^{\dagger}\right)\right] \\
& \left.+p^{2} g_{1 p} g_{2 p}\left[\bar{n}_{p}\left(\hat{\sigma}_{h 2}^{\dagger} \hat{\rho}_{S}(t) \hat{\sigma}_{h 1}+\hat{\sigma}_{h 1}^{\dagger} \hat{\rho}_{S}(t) \hat{\sigma}_{h 2}\right)\right]\right\} .
\end{aligned}
$$

The indicator $p$ here is redefined as $p=\frac{\omega_{a_{1,2} b}}{c}$. Introducing notations

$$
\gamma_{k l h}=\frac{2 p^{2} V_{p h t} g_{k p} g_{l p}}{\pi c}
$$

where $k, l=1,2, g_{k, l p}$ is the coupling constant corresponding to transition with frequency $\omega_{a_{1,2} b}$, and the average occupation numbers of photons $n_{h}=\bar{n}_{p}$, we rewrite Eq. A10 in a simple form as

$$
\begin{aligned}
\frac{d \hat{\rho}_{S}(t)}{d t}= & \sum_{k, l=1,2} \frac{\gamma_{k l h}}{2}\left[\left(n_{h}+1\right)\left(\sigma_{h l} \hat{\rho}_{S} \sigma_{h k}^{\dagger}+\sigma_{h k} \hat{\rho}_{S} \sigma_{h l}^{\dagger}-\sigma_{h l}^{\dagger} \sigma_{h k} \hat{\rho}_{S}-\hat{\rho}_{S} \sigma_{h k}^{\dagger} \sigma_{h l}\right)\right. \\
& \left.+n_{h}\left(\sigma_{h k}^{\dagger} \hat{\rho}_{S} \sigma_{h l}+\sigma_{h l}^{\dagger} \hat{\rho}_{S} \sigma_{h k}-\sigma_{h k} \sigma_{h l}^{\dagger} \hat{\rho}_{S}-\hat{\rho}_{S} \sigma_{h l} \sigma_{h k}^{\dagger}\right)\right] .
\end{aligned}
$$

The cross-couplings $\gamma_{k l h}$ for $k \neq l$ corresponds to the strength of the Fano interference, and $\gamma_{k k h}=\gamma_{k h}$ denotes the spontaneous decay rate. With this consideration, we finally obtain Eq. (3).

By the same procedure outlined for the transition $|b\rangle \leftrightarrow$ $\left|a_{1,2}\right\rangle$ induced by the high temperature photon reservoir (HTR), we easily obtain the Lindblad term describing the transition process $|b\rangle \leftrightarrow\left|\beta_{1,2}\right\rangle$ induced by the second low temperature reservoir (LTR2) in Eq. (4), where $\hat{\sigma}_{c k}=|b\rangle\left\langle\beta_{k}\right|$ $(k=1,2)$ is the lower operator. $n_{c 2}$ is the average phonon numbers of LTR2. The spontaneous decay rates and the crosscouplings are connected by,

$$
\Gamma_{k l c}=\frac{2 p^{2} V_{p h n} g_{k p} g_{l p}}{\pi c} .
$$

where the indicator $p$ is defined as $p=\frac{\omega_{\beta_{1,2} b}}{c}, g_{k, l p}$ is the coupling constant for transition with frequency $\omega_{\beta_{1,2} b} V_{p h n}$ is the phonon volume.

For the transition $\left|a_{1,2}\right\rangle \leftrightarrow\left|\alpha_{1,2}\right\rangle$ induced by the first low temperature phonon reservoir (LTR1), derivation is tedious but similar to the presented one. The complicity comes from the extra charge-separated states. The Hamiltonian describing the coupling of the system to the LTR1 in the interaction picture reads,

$$
\begin{aligned}
\hat{V}(t)= & \hbar \sum_{p} g_{11 p} \hat{\sigma}_{11}^{\dagger} \hat{b}_{p} e^{i\left(\omega_{a_{1} \alpha_{1}}-v_{p}\right) t}+\hbar \sum_{q} g_{12 q} \hat{\sigma}_{12}^{\dagger} \hat{b}_{q} e^{i\left(\omega_{a_{1} \alpha_{2}}-v_{q}\right) t} \\
& +\hbar \sum_{r} g_{21 r} \hat{\sigma}_{21}^{\dagger} \hat{b}_{r} e^{i\left(\omega_{a_{2} \alpha_{1}}-v_{r}\right) t}+\hbar \sum_{s} g_{22 s} \hat{\sigma}_{22}^{\dagger} \hat{b}_{s} e^{i\left(\omega_{a_{2} \alpha_{2}}-v_{s}\right) t}+\text { H.c.. }
\end{aligned}
$$

Following the same procedure summarized above, we obtain Eq. (5), where $\hat{\sigma}_{k l}=\left|\alpha_{k}\right\rangle\left\langle a_{l}\right|(k, l=1,2)$ or $\hat{\sigma}_{m n}=\left|\alpha_{m}\right\rangle\left\langle a_{n}\right|$
( $m, n=1,2)$ is the corresponding lower operator. $n_{c 1}$ is the average phonon numbers of LTR1. The spontaneous decay 
rates are

$$
\gamma_{k l m n}=\frac{2 p^{2} V_{p h n} g_{k l p} g_{m n p}}{\pi c} .
$$

with $V_{\text {phn }}$ the phonon volume. The indicator $p$ is defined as $p=\frac{\omega_{a_{i} \alpha_{i}}}{c}(i=1,2) . g_{k l, m n}$ is the coupling constant. $\gamma_{k l m n}(k l=m n)$ are the decay rates from the upper levels $\left|a_{1}\right\rangle$ and $\left|a_{2}\right\rangle$ to the lower levels $\left|\alpha_{1}\right\rangle$ and $\left|\alpha_{2}\right\rangle$, respectively. The cross-couplings $\gamma_{k l m n}(k l \neq m n)$ would lead to interferences.
As the involved levels (coupled to LTR1) here are more than those to HTR or LTR2, there are additional cross-couplings that do not exist in the case of HTR or LTR2. We obtain two types of cross-couplings in the case of LTR1. One is the same with that of HTR or LTR2, including $\gamma_{11,21}$ and $\gamma_{12,22}$. The other type includes $\gamma_{11,12}, \gamma_{11,22}, \gamma_{12,21}, \gamma_{21,22}$. The first type describes transitions to the same lower state, i.e., $\left|\alpha_{1}\right\rangle$ for $\gamma_{11,21}$ and $\left|\alpha_{2}\right\rangle$ for $\gamma_{12,22}$. But the second type is different.
[1] R. E. Blankenship, Molecular Mechanisms of Photosynthesis (Blackwell Science Ltd, Oxford, UK, 2002)

[2] H. van Amerongen, L. Valkunas, and R. van Grondelle, Photosynthetic Excitons (World Scientific, Singapore, 2000).

[3] T. Brixner, J. Stenger, H. M. Vaswani, M. Cho, R. E. Blankenship, and G. R. Fleming, Nature 434, 625 (2005).

[4] G. S. Engel, T.R. Calhoun, E. L. Read, T.-K. Ahn, T. Mančal, Y.-C. Cheng, R. E. Blankenship, and G. R. Fleming, Nature (London) 446, 782 (2007).

[5] T. R. Calhoun, N. S. Ginsberg, G. S. Schlau-Cohen, Y.-C. Cheng, M. Ballottari, R. Bassi, and G. R. Fleming, J. Phys. Chem. B 113, 16291 (2009).

[6] G. Panitchayangkoon, D. Hayes, K.A. Fransted, J. R. Caram, E. Harel, J. Wen, R. E. Blankenship, and G. S. Engel, Proc. Natl. Acad. Sci. U.S.A. 107, 12766 (2010).

[7] D. Hayes, G. Panitchayangkoon, K.A. Fransted, J. R. Caram, J. Wen, K. F. Freed, and G. S. Engel, New J. Phys. 12, 065042 (2010).

[8] E. Collini, C. Y. Wong, K. E. Wilk, P. M. G. Curmi, P. Brumer, and G. D. Scholes, Nature (London) 463, 644 (2010).

[9] E. Harel and G. S. Engel, Proc. Natl. Acad. Sci. U.S.A. 109, 706 (2012)

[10] M. B. Plenio and S. F. Huelga, New J. Phys. 10, 113019 (2008).

[11] M. Mohseni, P. Rebentrost, S. Lloyd, and A. Aspuru- Guzik, J. Chem. Phys. 129, 174106 (2008).

[12] A. Olaya-Castro, C. F. Lee, F. F. Olsen, and N. F. Johnson, Phys. Rev. B 78, 085115 (2008).

[13] A. Ishizaki and G. R. Fleming, Proc. Natl. Acad. Sci. USA 106, 17255 (2009).

[14] F. Caruso, A. W. Chin, A. Datta, S. F. Huelga, and M. B. Plenio, J. Chem. Phys. 131, 105106 (2009).

[15] P. Rebentrost, M. Mohseni, I. Kassal, S. Lloyd, and A. AspuruGuzik, New J. Phys. 11, 033003 (2009).

[16] G. D. Scholes, J. Phys. Chem. Lett. 1, 2 (2010).

[17] A. W. Chin, A. Datta, F. Caruso, S. F. Huelga, and M.B. Plenio, New J. Phys. 12, 065002 (2010).

[18] S. Lloyd and M. Mohseni, New J. Phys. 12, 075020 (2010).

[19] J. Strumpfer, M. Sener, and K. Schulten, J. Phys. Chem. Lett. 3, 536 (2012).

[20] H. Dong, D.-Z. Xu, J.-F. Huang, and C.-P. Sun, Light: Sci.
Appl. 1, e2 (2012).

[21] A. W. Chin, J. Prior, R. Rosenbach, F. Caycedo-Soler, S. F. Huelga, and M. B. Plenio, Nat. Phys. 9, 113 (2013).

[22] M. del Rey, A. W. Chin, S. F. Huelga, and M. B. Plenio, J. Phys. Chem. Lett. 4, 903 (2013).

[23] K. E. Dorfman, D. V. Voronine, S. Mukamel, and M. O. Scully, Proc. Natl. Acad. Sci. U.S.A. 110, 2746 (2013).

[24] B. P. Fingerhut, W. Zinth and R. de Vivie-Riedle, Phys. Chem. Chem. Phys. 12, 422 (2010).

[25] R. E. Blankenship, D. M. Tiede, J. Barber, G. W. Brudvig, G. Fleming, M. Ghirardi, M. R. Gunner, W. Junge, D. M. Kramer, A. Melis, T. A. Moore, C. C. Moser, D. G. Nocera, A. J. Nozik, D. R. Ort, W. W. Parson, R. C. Prince, and R. T. Sayre, Science 332, 805 (2011).

[26] M. O. Scully, K. R. Chapin, K. E. Dorfman, M. B. Kim, and A. Svidzinsky, Proc. Natl. Acad. Sci. U.S.A. 108, 15097 (2011).

[27] A. A. Svidzinsky, K. E. Dorfman, and M. O. Scully, Phys. Rev. A 84, 053818 (2011).

[28] C. Creatore, M. A. Parker, S. Emmott, and A. W. Chin, Phys. Rev. Lett. 111, 253601 (2013).

[29] V. Novoderezhkin, J. Dekker, and R. van Grondelle, Biophys. J. 93, 1293 (2007).

[30] E. Romero, I. H. M. van Stokkum, V. I. Novoderezhkin, J. P. Dekker and R. van Grondelle, Biochemistry 49, 4300 (2010).

[31] V. I. Novoderezhkin, E. Romero, J. P. Dekker, and R. van Grondelle, ChemPhysChem 12, 681 (2011).

[32] T. Cardona, A. Sedouda, N. Cox and A. W. Rutherford, Biochim Biophys Acta 181726 (2012).

[33] J.-L. Bredas, J. E. Norton, J. Cornil, and V. Coropceanu, Acc. Chem. Res. 42, 1691 (2009).

[34] Y. Y. Xu and J. Liu, Phys. Rev. E. 90, 052131 (2014).

[35] D. Abramavicius and S. Mukamel, J. Chem. Phys. 133, 184501 (2010).

[36] M. E. Madjet, A. Abdurahman, and T. Renger, J. Phys. Chem. B 110, 17268 (2006).

[37] D. Abramavicius and S. Mukamel, J. Chem. Phys. 133, 064510 (2010)

[38] M. O. Scully and M. S. Zubairy, Quantum Optics (Cambridge University Press, Cambridge, UK, 1997). 